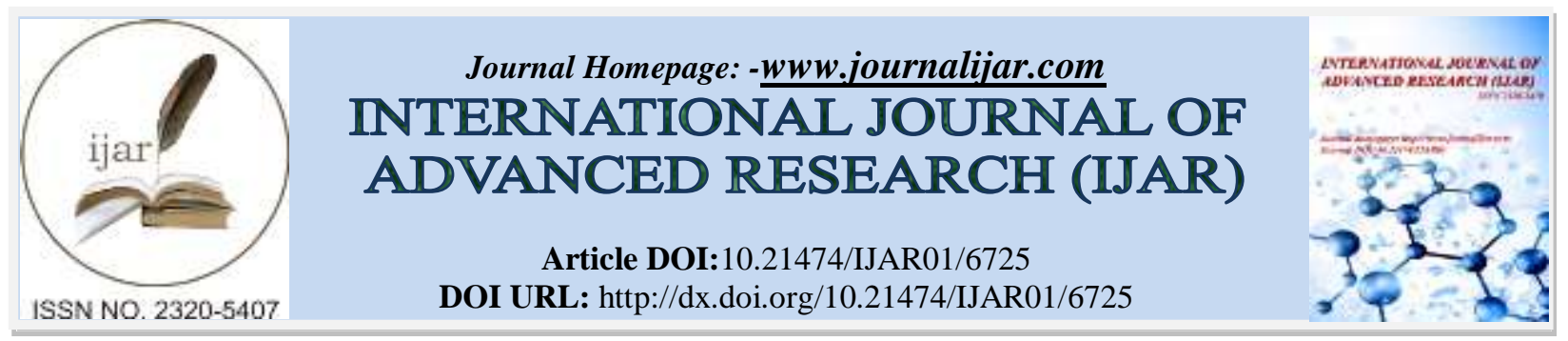

RESEARCH ARTICLE

\title{
SYNDROME DE FERNAND WIDAL: À PROPOS D'UNE OBSERVATION.
}

\author{
S. Ben elhend, H. Doulhousne, N. Hammoune, A. Mouhsine, E. Atmane and A. El fikri. \\ Service de Radiologie, Hôpital Militaire Avicenne, Marrakech. Maroc.
}

\section{Manuscript Info}

(..........................

Manuscript History

Received: 12 January 2018

Final Accepted: 14 February 2018

Published: March 2018

Keywords:-

Sinonasal polyposis - asthma - aspirin

intolerance.

\section{Abstract}

The Fernand Widal syndrome is a rare disease that associates a chronic rhinosinusitis with sino-nasal polyposis, asthma and a clinical intolerance of aspirin. Through this observation we describe the different signs and therapeutic aspects of this unrecognized disease usually diagnosed late.

\section{Introduction:-}

La maladie de Fernand Widal est une affection rare dont le diagnostic - le plus souvent tardif - repose sur la triade polypose naso-sinusienne, asthme et intolérance à l'acide acétylsalicylique. L'imagerie est non spécifique et permet d'apprécier l'atteinte naso-sinusienne.

\section{Observation :}

Il s'agissait d'un homme de 46 ans, asthmatique, suivi pour polypose nasale (opéré à deux reprises) avec notion d'allergie à l'aspirine, qui rapportait une obstruction avec tuméfaction nasale, des rhinorrhées associées à un prurit et éternuements. L'examen par endoscopie nasale montrait une obstruction nasale avec des polypes bilatéraux. Le complément TDM avait mis en évidence un comblement tissulaire total des cavités sinusales et des fosses nasales (figures : A, B et C).

\section{Discussion:-}

Le syndrome de FERNAND WIDAL est une entité nosologique - décrite pour la première fois en 1929 par F. Widal- associant une polypose naso-sinusienne, un asthme et une intolérance à l'acide acétylsalicylique [1]. Sa physiopathologie n'est pas encore complètement clarifiée et sa prévalence demeure incertaine. Il survient typiquement chez l'adulte entre 40 et 50 ans. Le diagnostic est en général facile dès l'interrogatoire dans les formes complètes. Cependant le tableau peut être incomplet au début, prédominé par les signes de la sphère ORL, conduisant au diagnostic d'une rhinite non allergique. L'asthme survient le plus souvent 2 à 3 ans plus tard, sévère et précède généralement la polypose nasale dans $2 / 3$ des cas. L'intolérance aux AINS dont l'aspirine entraine des bronchospasmes plus ou moins sévères. L'intensité de la symptomatologie varie de la simple rhinite jusqu'à des manifestations anaphylactoïdes mettant en jeu le pronostic vital. Son diagnostic repose sur le test de provocation orale qui consiste à administrer des quantités croissantes d'aspirine toutes les 30 minutes. Une chute d'au moins $20 \%$ du VEMS ou l'apparition de symptômes extra-thoraciques (rhinorrhée, érythème, conjonctivite) permet de considérer le test comme positif. Il doit être réservé à des patients avec un asthme stabilisé sans antécédents de crises sévères suite à la prise d'AINS, de pathologie sous-jacente sévère, d'infection respiratoire récente, de grossesse en cours, ni de prise de bêtabloquants. Les corticoïdes et les bronchodilatateurs doivent être autant que possible réduits, 
voire supprimés pour éviter les faux négatifs [2-3]. La présence d'un asthme intolérant à l'aspirine doit fortement faire évoquer le diagnostic.

La polypose naso-sinusienne (PNS) est une forme particulière des sinusites chroniques, caractérisée par le développement bilatéral et multifocal de polypes dont le diagnostic repose sur l'imagerie en montrant un comblement tissulaire plus ou moins marqué des cavités naso-sinusiennes [1].

La corticothérapie locale continue et prolongée est le traitement de référence de la PNS. La chirurgie est indiquée en cas PNS invalidante et résistante à un traitement médical bien conduit, bien observé et suffisamment prolongé (polypectomie, ethmoïdectomie) [1].

\section{Conclusion:-}

Le diagnostic du syndrome de Widal repose sur la triade asthme, polypose nasale et intolérance à l'aspirine ou aux AINS. La prescription de ces médicaments chez ces patients doit être prudente car les réactions provoquées peuvent mettre en péril le pronostic vital en l'absence d'une prise en charge urgente.

\section{Déclarations d'intérêts:}

Les auteurs déclarent ne pas avoir aucun conflit d'intérêts avec ce manuscrit.

\section{Figures:-}
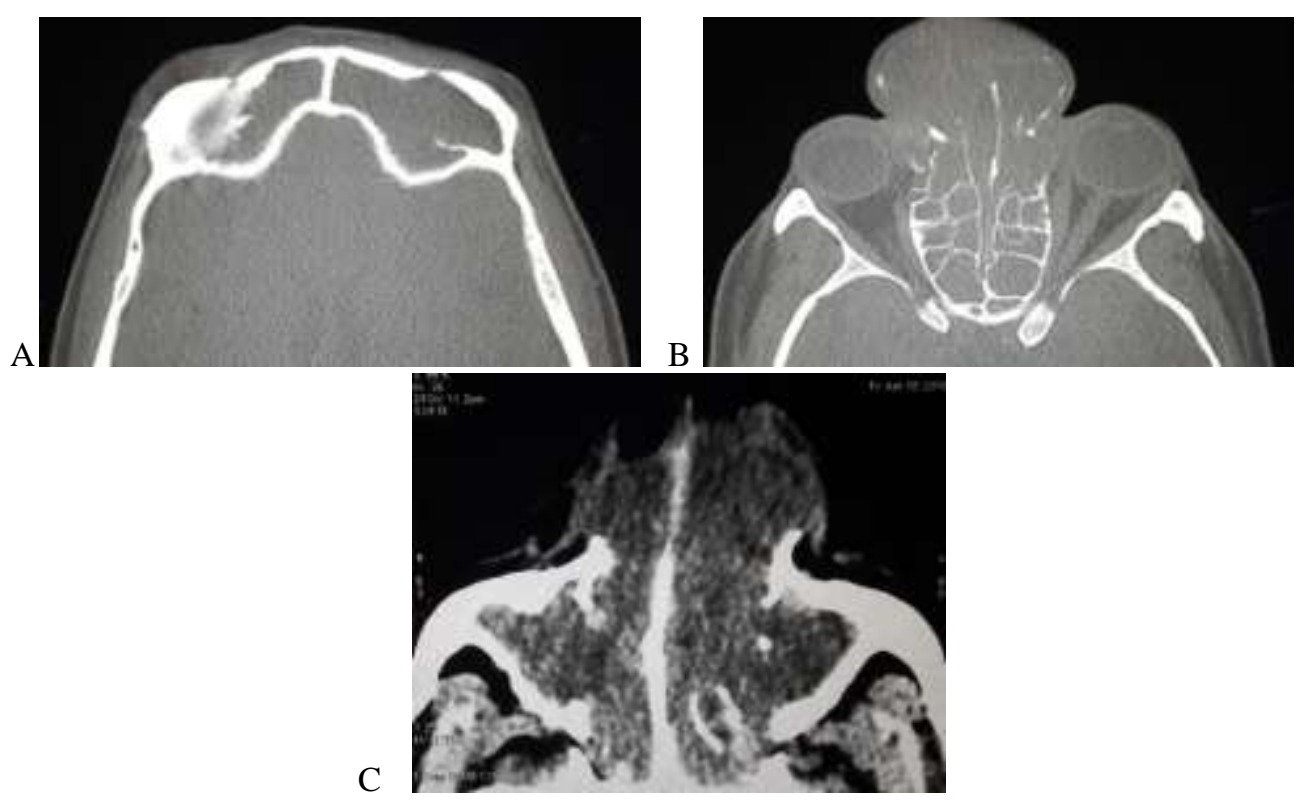

Figues (A et B: Fenêtre osseuse et C: Fenêtre parenchymateuse) :

Hypertrophie nasale avec comblement tissulaire total des cavités sinusales et des fosses nasals et amincissement de la charpente osseuse.

\section{Bibliographie:-}

1. Serrano E, Wessel F. La maladie de Fernand-Widal. Diagnostic et traitement. Revue Française d'Allergologie et d'immunologie clinique 43 (2003) 135-137.

2. Boumezaoued $\mathrm{S}$, Amara $\mathrm{B}$, Serraj $\mathrm{M}$. Asthme avec intolérance à l'aspirine : à propos de 21 cas. Revue Française d'Allergologie et d'immunologie clinique 52 (2012) 293-297.

3. Bourrain J.L. Faut-il et comment explorer l'asthme à l'aspirine ? Revue Française d'Allergologie et d'immunologie clinique 51 (2011) 227-229. 